\title{
Erratum to: Genetic variation in the carbonyl reductase 3 gene confers risk of type 2 diabetes and insulin resistance: a potential regulator of adipogenesis
}

\author{
Yi-Cheng Chang • Pi-Hua Liu • Yun-Chih Tsai • Yen-Feng Chiu • Shyang-Rong Shih • Low-Tone Ho • Wen-Jane Lee • \\ Chieh-Hua Lu • Thomas Quertermous • J. David Curb • Wei-Jei Lee • Po-Chu Lee • You-Han He • Jih-I Yeh • \\ Juey-Jen Hwang • Shu-Huei Tsai • Lee-Ming Chuang
}

Published online: 31 May 2012

(C) Springer-Verlag 2012

Erratum to: J Mol Med

DOI 10.1007/s00109-012-0898-8

There is a mistake in the original publication. The affiliation for the author Pi-Hua Liu is incorrect.

The online version of the original article can be found at http://dx.doi.org/ 10.1007/s00109-012-0898-8.

Y.-C. Chang • Y.-C. Tsai · S.-R. Shih · J.-J. Hwang • S.-H. Tsai •

L.-M. Chuang $(\square)$

Department of Internal Medicine,

National Taiwan University Hospital, 7 Chung-Shan South Road,

Taipei, Taiwan

e-mail: leeming@ntu.edu.tw

Y.-C. Chang

Genomics Research Center, Academia Sinica, Taipei, Taiwan

P.-H. Liu

Clinical Informatics and Medical Statistics Research Center,

Chang Gung University, Taoyuan, Taiwan

Y.-F. Chiu

Division of Biostatistics and Bioinformatics, Institute of

Population Health Sciences, National Health Research Institutes,

Zhunan, Taiwan

L.-T. Ho

Department of Medical Research and Education,

Taipei Veterans General Hospital, Taipei, Taiwan

L.-T. Ho

Section of Endocrinology and Metabolism,

Department of Medicine,

Taipei Veterans General Hospital, Taipei, Taiwan

L.-T. Ho

Faculty of Medicine, School of Medicine, National Yang-Ming University, Taipei, Taiwan

W.-J. Lee

Department of Medical Research, Taichung Veterans General

Hospital, Taichung, Taiwan
The correct affiliation is: Clinical Informatics and Medical Statistics Research Center, Chang Gung University, Taoyuan, Taiwan.

C.-H. Lu

Division of Endocrinology and Metabolism,

Tri-Service General Hospital,

Taipei, Taiwan

T. Quertermous

Division of Cardiovascular Medicine, Falk Cardiovascular, Research Building, Stanford University School of Medicine, Stanford, CA, USA

\section{J. D. Curb}

John A. Burns School of Medicine, University of Hawaii, Honolulu, HI, USA

\section{W.-J. Lee}

Department of Surgery, Ming-Sheng General Hospital,

Taoyuan, Taiwan

P.-C. Lee

Department of General Surgery,

National Taiwan University Hospital,

Taipei, Taiwan

Y.-H. He

Department of Laboratory Medicine, National Taiwan University Hospital Yunlin Branch, Doaliao, Taiwan

J.-I. Yeh

Department of Family Medicine,

Buddhist Tzu Chi General Hospital,

Hualien', Taiwan

L.-M. Chuang

Graduate Institute of Clinical Medicine, College of Medicine,

National Taiwan University, Taipei, Taiwan 